\title{
Reimagining the Visceral Protective Layer with Tailored Manipulation: A Case Report
}

\section{R. Dewayne Edwards', Luis G. Fernandez², Sean 0'Keefe ${ }^{3}$, Michelle Baribault ${ }^{4}$, Marc R. Matthews ${ }^{5 *}$}

\author{
${ }^{1}$ Burn Surgery and Surgical Critical Care, The Arizona Burn Center, Phoenix, AZ, USA \\ ${ }^{2}$ Department of Surgery, University of Texas Health Science Center, Tyler, TX, USA \\ ${ }^{3} 3 \mathrm{M}$, Phoenix, AZ, USA \\ ${ }^{4}$ Mountain Vista Medical Center, Mesa, AZ, USA \\ ${ }^{5}$ The Arizona Burn Center, Phoenix, AZ, USA \\ Email: dnrme44@hotmail.com, thebigkahuna115@gmail.com, sokeefe2@mmm.com, michelle.baribault@steward.org, \\ *marc_matthews@dmgaz.org
}

How to cite this paper: Edwards, R.D. Fernandez, L.G., O'Keefe, S., Baribault, M. and Matthews, M.R. (2022) Reimagining the Visceral Protective Layer with Tailored Manipulation: A Case Report. Surgical Science, 13, 53-65.

https://doi.org/10.4236/ss.2022.132009

Received: November 24, 2021

Accepted: January 25, 2022

Published: January 28, 2022

Copyright $\odot 2022$ by author(s) and Scientific Research Publishing Inc. This work is licensed under the Creative Commons Attribution International License (CC BY 4.0).

http://creativecommons.org/licenses/by/4.0/

\begin{abstract}
The visceral protective layer is a standard component of the ABTHERA ${ }^{\mathrm{TM}}$ systems for temporary abdominal closures. Nonetheless, there are circumstances where the standard, fenestrated visceral protective layer is too large to be successfully applied into every patient's open abdomen, such as within the abdomen of a child, smaller adult or a patient with previously placed ostomies or drains. The fenestrated, visceral protective layer may require alterations or tailoring for adequate deployment instead of placing the bulk of the visceral protective layer entirely into the open abdomen for temporary abdominal closure. This case report illustrates how the visceral protective layer can be adapted or "reimagined" to conform to a patient with unique or complex abdominal domain features when utilizing the ABTHERA ${ }^{\mathrm{TM}}$ device prior to facial closure or abdominal wall reconstruction. Photographs are utilized in a step-by-step fashion to aid the clinician in these detailed maneuvers.
\end{abstract}

\section{Keywords}

Open Abdomen, Temporary Abdominal Closure, Visceral Protective Layer, Loss of Abdominal Domain

\section{Introduction}

Since the 1990's, the negative pressure wound therapy (NPWT) devices have been widely used in the patient requiring a temporary abdominal closure (TAC) to rapidly evacuate wound debris, exudate, and prokaryotic organisms or to simply 
release an abdominal compartment syndrome. Though these commercial devices are relatively new to surgical management, the concept of negative pressure wound therapy can be traced back to the Roman Empire [1]. The development and use of a polyurethane foam coupled with a mechanical vacuum was pioneered by Dr. Louis Argenta and Dr. Michael Morykwas at Wake Forest University School of Medicine [2] [3]. The NPWT devices have proven to be extremely effective and advantageous in the healing of both routine and complex surgical wounds and have been helpful in open abdominal wounds that are scheduled for a planned "second look" operation or abdominal washout [3].

The ABTHERA ${ }^{\mathrm{TM}}$ device (3M, Minneapolis, $\mathrm{MN}$ ) was further developed by the forward-thinking and highly innovative KCI company in San Antonio, TX [2]. Within the ABTHERA ${ }^{\mathrm{TM}}$ device system is the visceral protective layer (VPL) (3M, Minneapolis, $\mathrm{MN}$ ), an invaluable component that helps protect the viscera and assists with the removal of fluid and debris through fenestrations in the VPL. The effluent is subsequently displaced by negative pressure via the VAC Ulta device through a separate foam layer and then tubing for final disposal into a canister [2] [3] [5]. Not only does the VPL protect the abdominal viscera but separates the bowel from the anterior abdominal wall preventing the development of adhesions that may hinder rapid or repeated re-entrance into the abdominal cavity [4] [5] [6] [7].

The fenestrated VPL is simply designed with a flexible, perforated, polyurethane drape measuring $665 \mathrm{~mm} \times 8022 \mathrm{~mm}$ that encases blue foam segments with a central body and six primary, extension limbs all connected in a block format [4] [5] [6] [7] (Figure 1(a) and Figure 1(b)). The encapsulated foam measures $10 \mathrm{~mm}$ in thickness [4] [5] [6] [7]. Modifications of the ABTHERA ${ }^{\mathrm{TM}}$ and the ABTHERA ADVANCE ${ }^{\mathrm{TM}}$ Open Abdomen Dressing have been previously described by various authors, in the literature [3] [8] [9] [10] [11] [12]. One of the challenges of using the prefabricated VPL is that a "one size fits all" device is sometimes not practical. The device's use may be limited by the patient's size, such as in the pediatric population, or the presence of drains or ostomies. Other factors such as patient domain limitations in smaller adults or in those with partial fascial closure, may restrict the usefulness of the nonmodified VPL due to its larger size where simply folding over of the VPL may not be the best answer or practical [5]. Therefore, it might be better to describe the VPL as "one size fits many, but not all". Smaller adults, for example, under 5 foot 4 inches have a concomitant smaller abdominal domain and may not be able to accommodate the standard VPL tucked over the liver and down into the paracolic gutters and pelvis while left folded in place [4] [5].

Fortunately, the VPL may be circumferentially modified so that the outer edges of the fenestrated VPL with its encased foam will fit comfortably into the patient's abdomen. A key requirement, that must be met in any VPL modification is ensuring that no sponge is exposed beyond the polyurethane barrier layer of the VPL [5]. VPL modification may be necessary to fit the device into the abdominal cavity and thereby still allowing for bowel protection. Furthermore, patients 


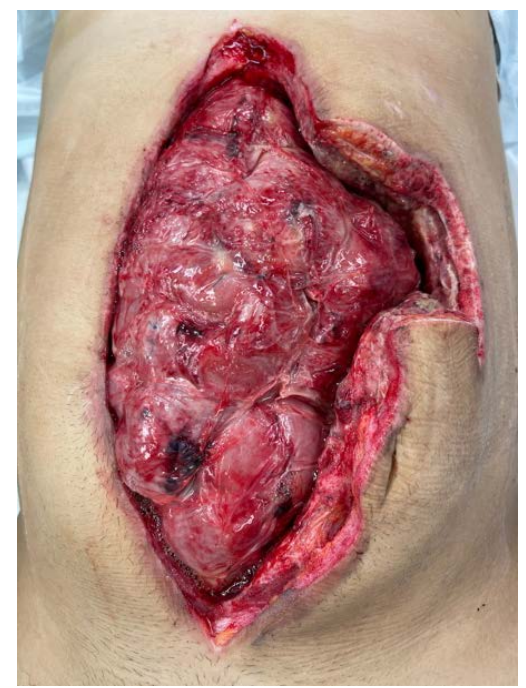

(a)

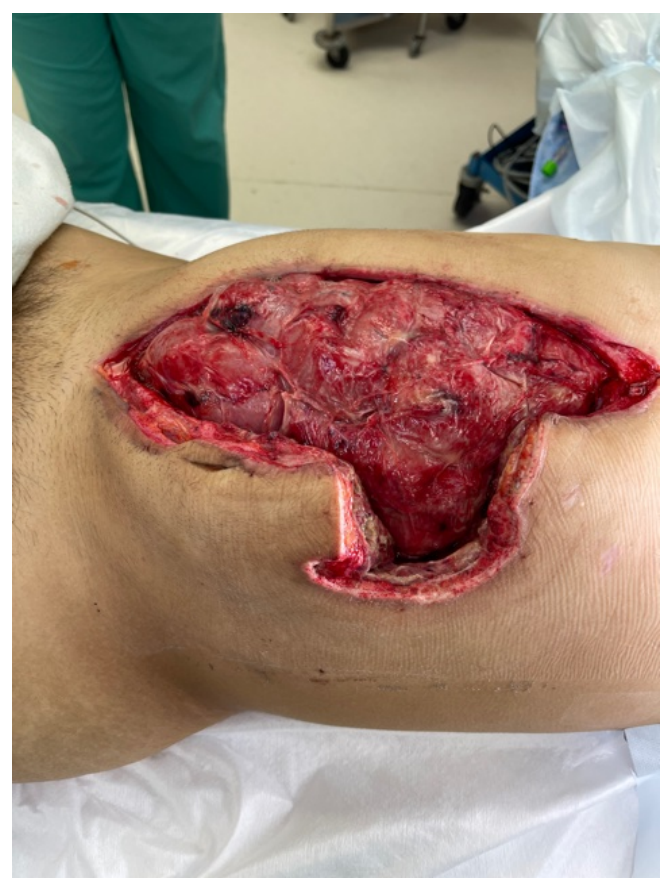

(b)

Figure 1. (a) Photograph of the anterior, open abdomen that has a well-cocooned small and large bowel with a left lateral, open, former ostomy wound site after colonic reanastomosis. (b) Photograph of the open abdomen (left lateral view) that has a wellcocooned small and large bowel with a left lateral, open, former ostomy wound site after colonic re-anastomosis.

with internal abdominal adhesions eventually may form a well-cocooned, small and large bowel visceral mass that may not accommodate the large size of the standard VPL. Adhesions between the bowel and the abdominal wall may require surgical adhesiolysis prior to VPL placement; however, such a procedure also increases the risk for an enterotomy and possible fistula formation. Depending on the size of the child, small adult or the added complexity of the ab- 
domen (intraabdominal adhesions, ostomies, etc.), the VPL can be altered to the patient's abdominal domain.

One option is to use a "limb" or "leg" of the blue, blocked foam encased in the fenestrated VPL's polyurethane layers, with or without a portion of the VPL's main foam body. While this exposes one edge of foam to the bowel, that portion of exposed foam can be easily removed with scissors, leaving a long linear and narrow polyurethane cuff that is as effective in protecting the bowel from the foam. Furthermore, the central portion of the VPL may be used if tailored correctly, but the overall configuration must fit comfortably in the abdominal cavity. No matter the configuration utilized, the tailored or reimagined VPL would allow negative pressure to sweep fluid, pro-inflammatory mediators, prokaryotic organisms such as bacteria, and necrotic debris from the abdominal cavity as originally described. This case report was found to be exempt by the institutional review board at Maricopa Medical Center/Valleywise Medical Center protocol number 2021-037 according to 45CFR46.104(9).

\section{Case Report}

A 20-year-old male (height of 5 feet 6 inches $(168 \mathrm{~cm})$, weight of $131 \mathrm{lb}$. $(59.8$ $\mathrm{kg}$ ), basal metabolic index of $21.2 \mathrm{~kg} / \mathrm{m}^{2}$ ), status post accidental gunshot wound to the abdomen seven months prior, had initially undergone an exploratory laparotomy with a segmental resection of his transverse colon, primary repair of his gastric injury, partial omentectomy, splenectomy, and evacuation of the hemoperitoneum. On postoperative day 2, the patient was re-explored in the operating room (OR) for a planned second look laparotomy and underwent further resection of his omentum with mobilization of the colon's splenic flexure. An end colostomy was developed, as well as the creation of a mucous fistula. Seven months later he presented for reversal of his colostomy. He underwent a takedown of the colostomy, including a hand-sewn, colo-colonic anastomosis. Noted intraoperatively, the small bowel and remaining large bowel were densely cocooned with adhesions. In addition, there were thick adhesions between the viscera and the abdominal wall (Figure 1(a) and Figure 1(b)) requiring some anterior abdominal wall to viscera adhesiolysis for several centimeters from the midline laparotomy incision before colonic re-anastomosis creation and fascial closure.

On post-operative day two, the patient was noted to develop tachycardia and hypotension coupled with a drop in his hemoglobin to $5 \mathrm{gm} / \mathrm{dL}$ an abdominal computerized tomography revealed a large fluid collection and arterial blush consistent with a hematoma immediately adjacent to the mesentery and newly created anastomosis. After adequate volume resuscitation the patient returned emergently to the OR for re-exploration where a large hematoma was evacuated, and a small mesenteric bleeding vessel was ligated. The old colostomy site was reinspected and debrided as a portion of its anterior abdominal wall subcutaneous tissue and skin had become necrotic. 


\section{Procedure in Detail}

The patient had an intrabdominal VPL device (Figure 2) deployed, cut with scissors in an elliptical pattern with removal of 2.5 blue foam blocks encases in polyurethane and applied over the intraabdominal viscera (Figure 3 ) as the standard VPL was too large in circumference to fit into the abdomen cavity as

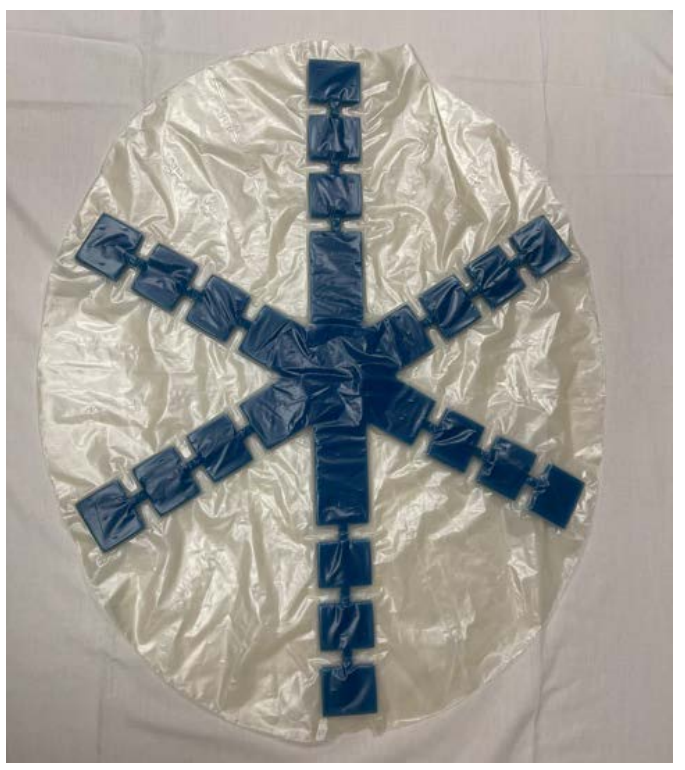

Figure 2. Photograph of a standard, unaltered VPL device with polyurethane drape encasing the blue foam.

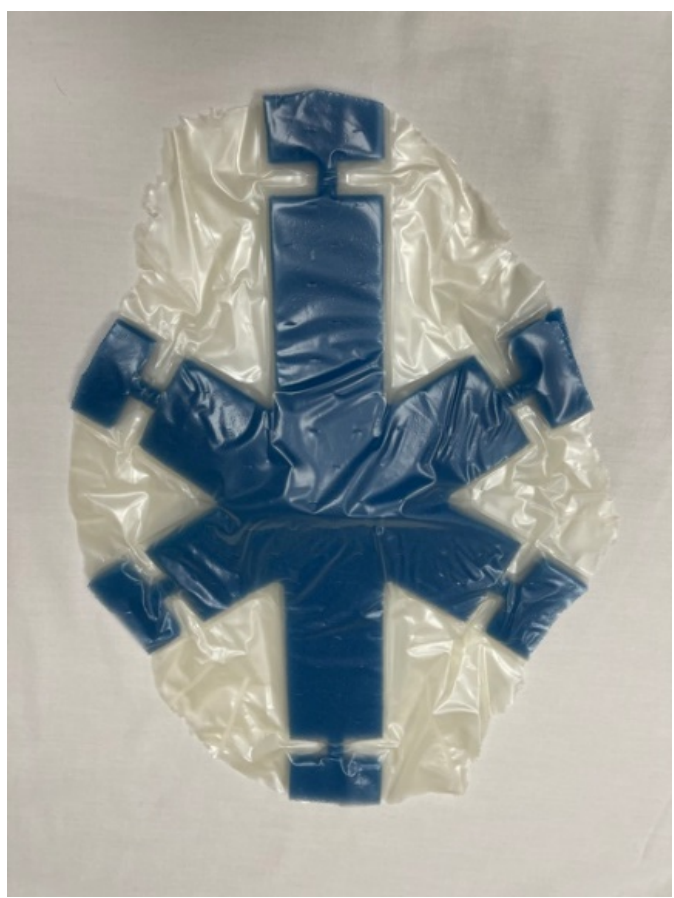

Figure 3. Photograph of a tailored VPL in an elliptical shape with removal of 2.5 blue foam blocks encased in polyurethane. Note the half block remaining would be in direct contact with the viscera without manual removal. 
the result of dense adhesions. With the plan for a second look operation within 24-hours, the VPL was furthered tailored to remove the exposed blue foam tabs (Figure 4(a) and Figure 4(b)). There was minimal surface exposure to tuck the entire VPL under the abdominal wall and over the bowel to afford adequate visceral protection without a concomitant, large adhesiolysis and its inherent risks. The modified VPL tailored in an elliptical shape was tucked (Figure 5) into the

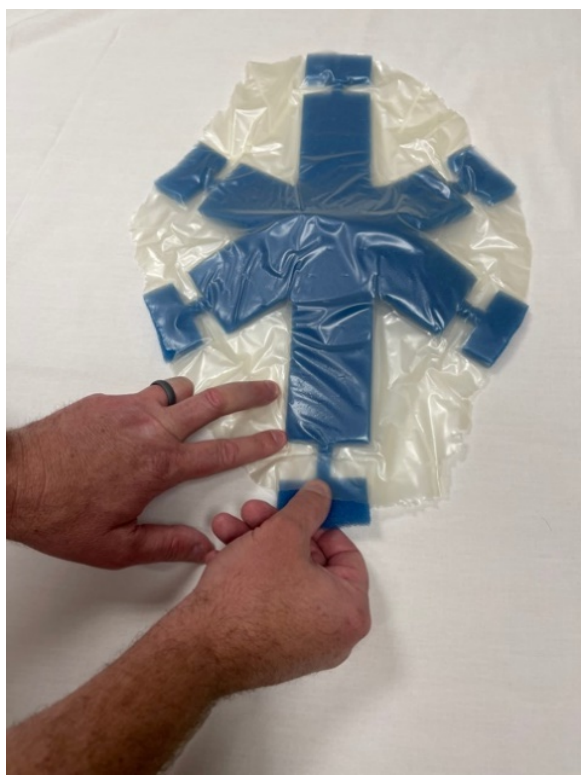

(a)

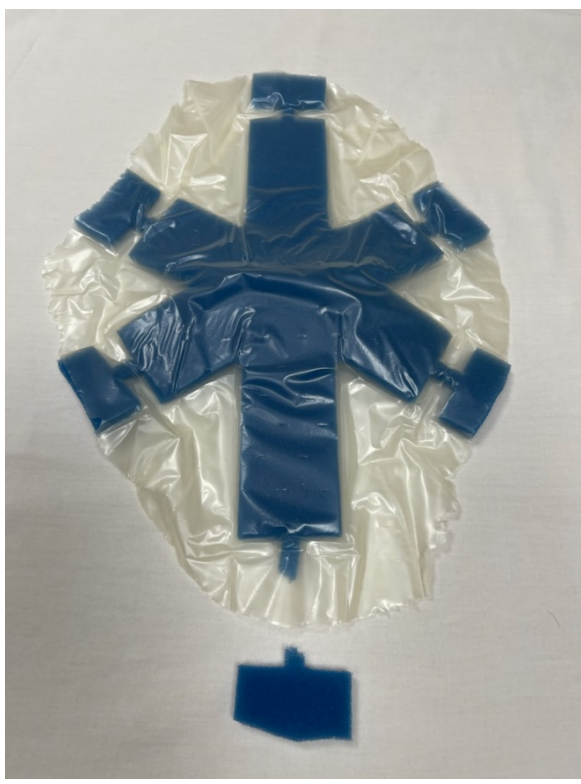

(b)

Figure 4. (a) Photograph of a tailored VPL in an elliptical shape with removal of 2.5 blue foam blocks encased in polyurethane. Note the half block remaining is being manually removed so as not to be in contact with the viscera; (b) Photograph of a tailored VPL in an elliptical shape with removal of the remaining foam block so as not to be in contact with the viscera. 


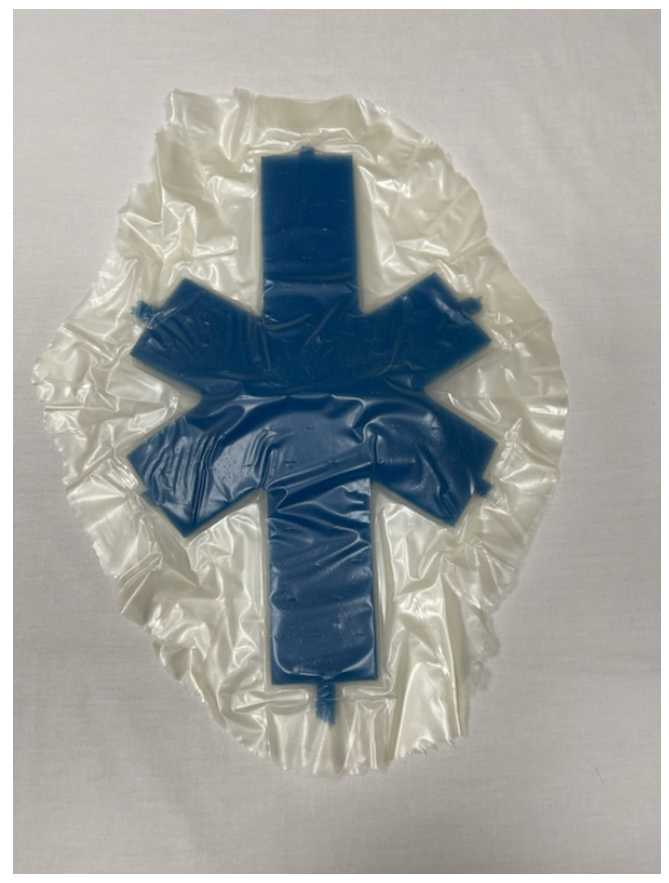

Figure 5. Photograph of a tailored VPL polyurethane drape without exposed blue foam that was used with the first operation requiring a TAC.

abdominal cavity under the eaves of the abdominal wall to protect the underlying viscera. The ABTHERA ADVANCE ${ }^{\mathrm{TM}}$ fenestrated blue foam was placed over the VPL, followed by acrylic drapes at the skin level, followed by a SensaT.R.A.C pad to deliver $-125 \mathrm{mmHg}$ negative pressure via the VAC Ulta Device.

The patient returned to the OR where proximal and distal aspects of the midline facia were sutured together for a partial fascial closure. This was done to preserve fascial domain for eventual closure at a subsequent date. This partial closure created difficulty in providing a TAC with the ABTHERA ${ }^{\mathrm{TM}}$ using the standard, unmodified VPL. Therefore, the decision was made to modify the VPL again, so that it would cover the exposed viscera followed by sponge application and drape placement. Using only one limb of the fenestrated VPL and part of the VPL's central blue sponge (Figure 6(a) and Figure 6(b)) with the polyurethane drape, was modified to fit the open abdomen. This was done in a way that also protected the underlying bowel from any exposed sponge. Because of bowel edema, loss of domain, and the threat of possible contact with the exposed viscera, the remaining central portion of the VPL's foam (a portion of the central foam body) was removed from the polyurethane drape leaving the remaining foam well-covered by the remaining polyurethane (Figures 7(a)-(c)). This modified or reimagined "wedge-shaped" VPL was then tucked into the patient's abdominal cavity to protect the bowel but under (Figure 8(a) and Figure 8(b)) followed by the standard ABTHERA ${ }^{\mathrm{TM}}$ sponge, acrylic drape and SensaT.R.A.C pad (Figure 9). Approximately 48 hours later, and following significant diuresis, the patient had completion of his abdominal, fascial closure and portions of the skin with placement of a standard NPWT at the skin level. The patient was 


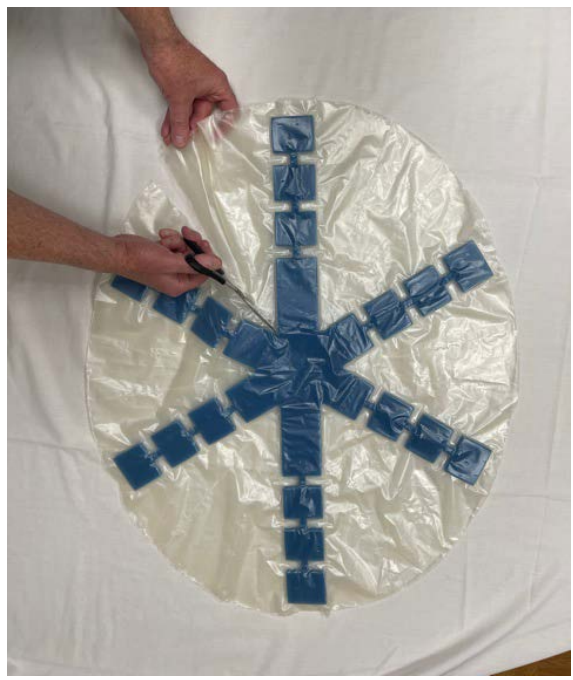

(a)

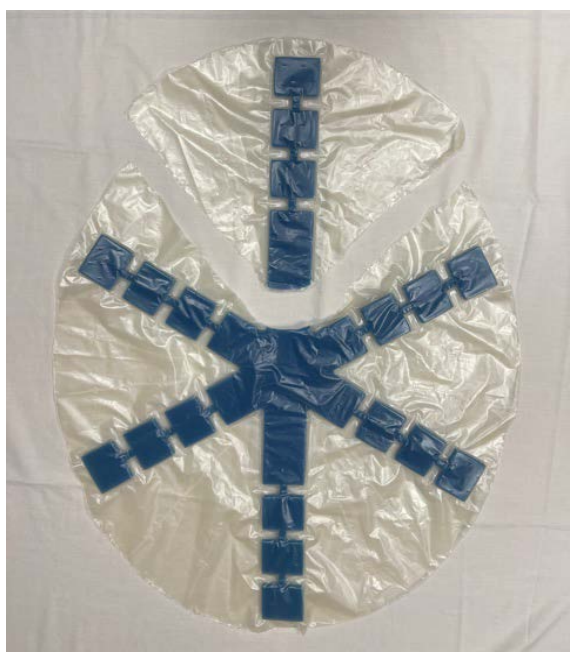

(b)

Figure 6. (a) Photograph of the active tailoring of the VPL to achieve a wedge-shaped configuration for intraabdominal placement; (b) Photograph of the VPL with the completed wedge-shaped separation of the VPL from the rest of the larger VPL.

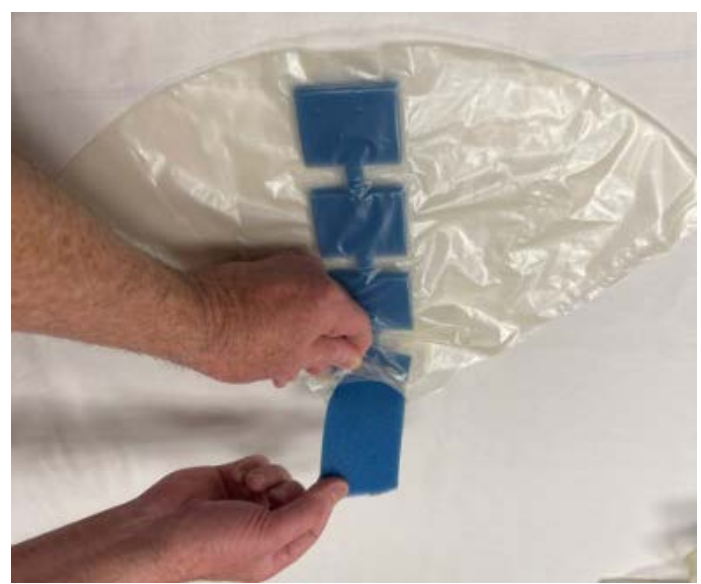

(a) 


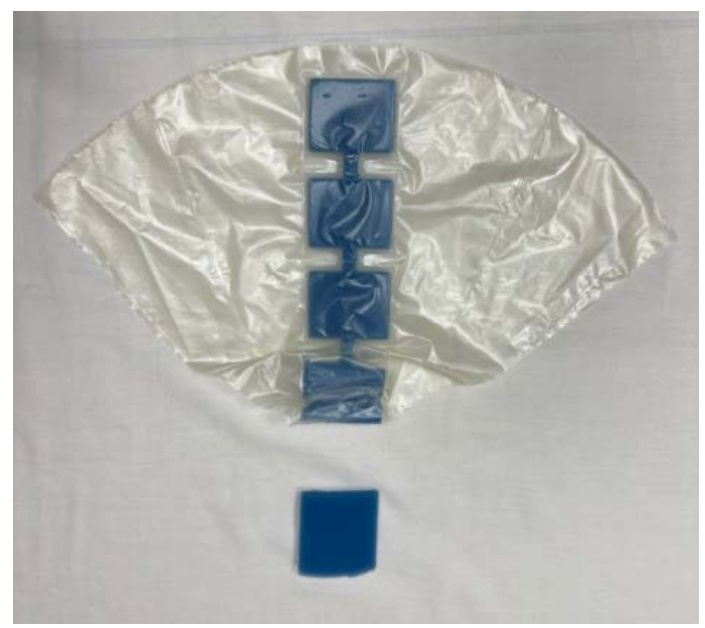

(b)

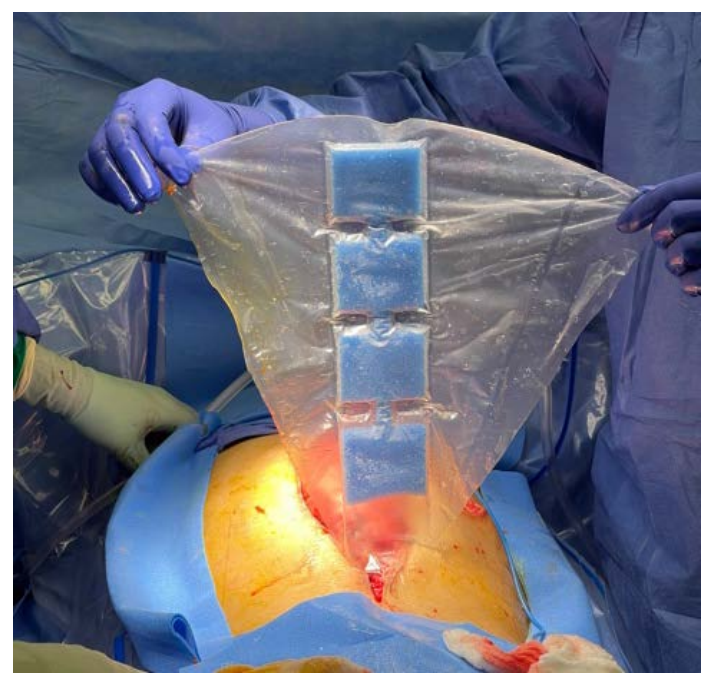

(c)

Figure 7. (a) Manual removal of a portion of the VPL blue foam sponge from the VPL dressing; (b) Tailored removal of a portion of the VPL blue foam sponge to prevent sponge exposure to the bowel; (c) Photograph of the wedged-shaped visceral protective layer modified using scissors ready to be placed into the abdominal cavity to protect the viscera and evacuate debris, bacteria, and pro-inflammatory mediators.

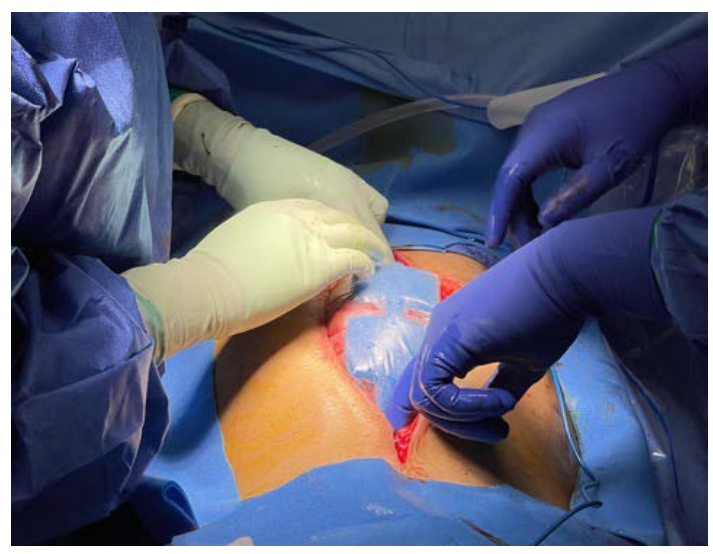

(a) 


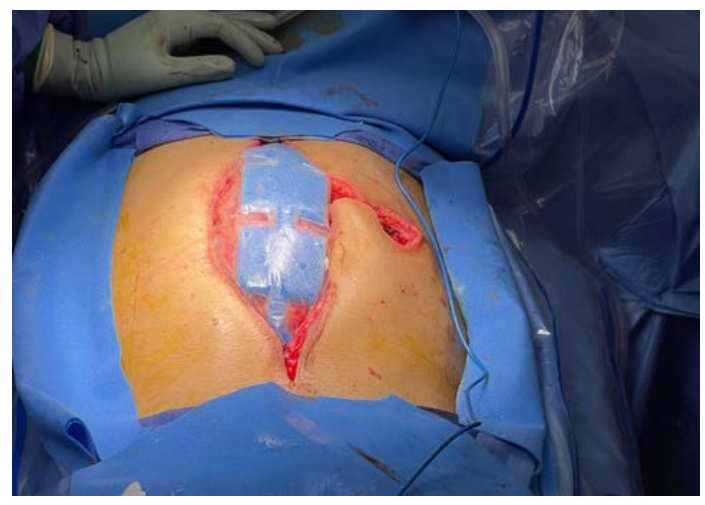

(b)

Figure 8. (a) Photograph of the insertion of the modified visceral protective layer into the abdomen with viscera protection prior to application of the foam sponge and acrylic drapes; (b) Photograph of the final insertion of the modified visceral protective layer into the abdomen with viscera protection prior to application of the foam sponge and acrylic drapes.

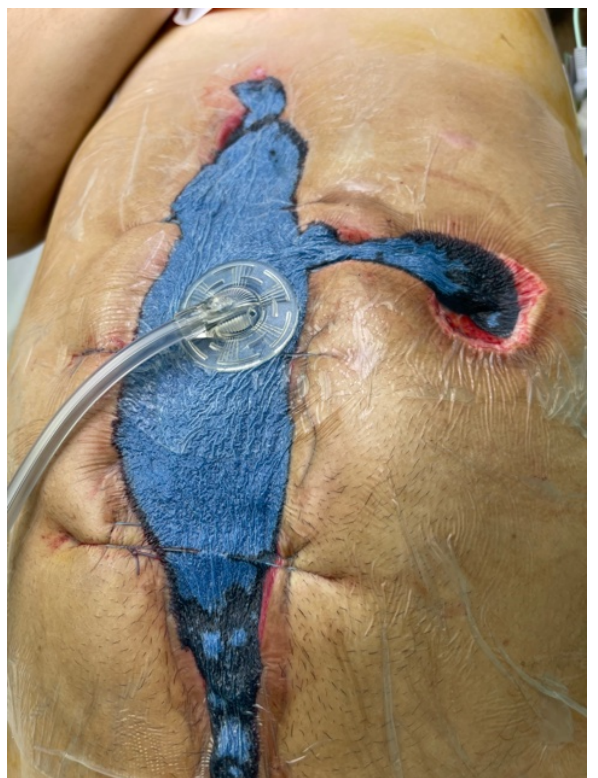

Figure 9. Photograph of the application of the ABTEHRA ADVANCE blue foam sponge with visible retention sutures covered with an acrylic drape and followed by application of a SensaT.R.A.C pad.

eventually discharged with follow-up in the trauma clinic tolerating a diet (Figure 10(a) and Figure 10(b)). Unfortunately, further complications occurred and future nonoperative plans are being developed.

\section{Discussion}

The fenestrated VPL is a well-engineered and designed intra-abdominal device to protect the large and small bowel while removing excess intrabdominal fluid, debris, bacteria and pro-inflammatory mediators during TAC. It also provides another layer in preventing the outside environment from contaminating the 


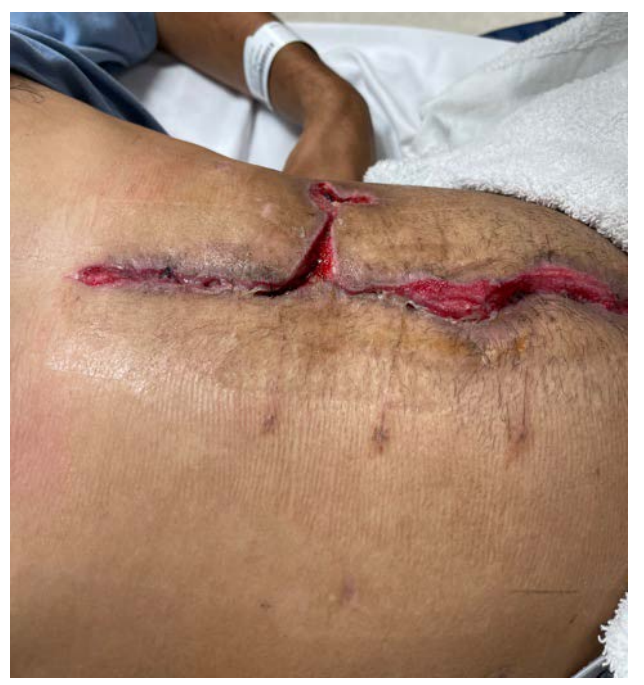

(a)

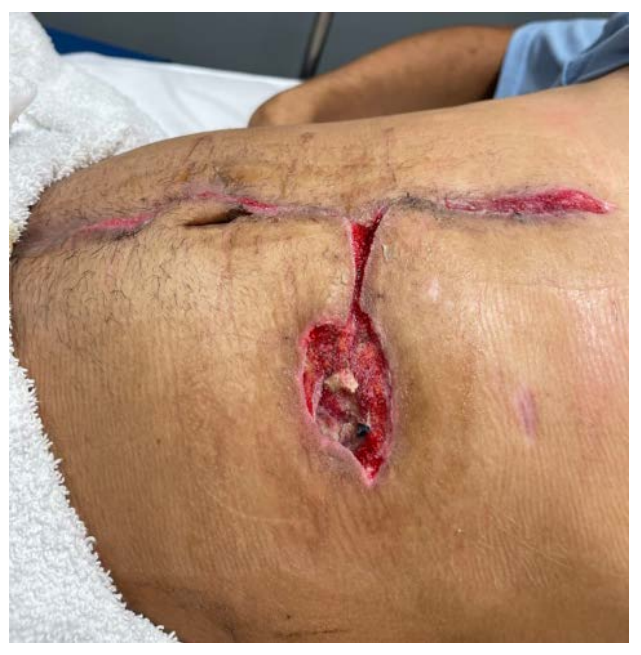

(b)

Figure 10. Right and left lateral projections of the patient's abdominal wall status-post fascial closure on the first clinic follow-up with dressings removed.

abdominal cavity [2] [5]. Coupled with the ABTEHRA ADVANCE ${ }^{\mathrm{TM}}$ device (including its blue foam, fenestrated sponges and the acrylic drapes), the negative pressure system is easily deployed for standard to large sized individuals with no previous history of abdominal interventions. However, since "one-size does not fit all”, many individual patients with prior abdominal surgery require VPL tailoring prior to insertion into the intra-abdominal compartment. The fenestrated VPL size can be altered to fit the patient's inherent abdominal configuration including the size of the patient (adult or child), presence of intra-abdominal adhesions, presence of a prominent falciform ligament, hepatomegaly, edematous bowel, presence of ostomies, or after drain placement through the anterior abdominal wall. Stating that the excess VPL can just "fold over" the surgeon's hand as mentioned in the ABTHERA ${ }^{\mathrm{TM}}$ System instruction guide, is no longer sufficient without at least discussing the need for tailoring or reimagining the VPL 


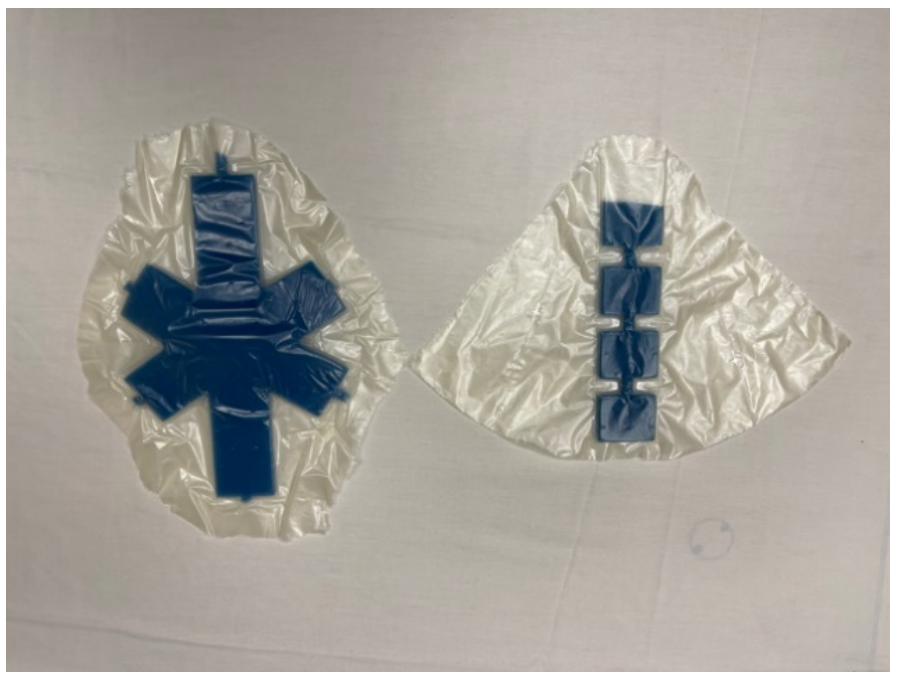

Figure 11. Photograph of the two types of VPL modifications for a side by side comparison. The left modified VPL is in an elliptical pattern while the right modifird VPL is a wedge-shaped pattern. Either VPL shape can be utilized based on the speficiations required by the surgeon in fitting the modified VPL into the abdomen intraoperatively.

[5]. The "preferred method" is no longer believed to be just folding over the VPL unto itself [5]. This adds unnecessary filler to the open abdomen cavity and could be problematic for the abdomen opened for abdominal compartment syndrome. The actual "preferred method" should be cutting and discarding the excess VP. It is routine for surgeons to discerningly excise diseased human tissue during an operation. Such is the same with these inanimate objects used for TAC. It would be a rarity for the surgeon to cut the VPL "too short" [5]. Tailoring of the VPL falls in the domain of the mastery of surgery.

In this case report, various VPL shapes were utilized including an elliptical configuration and a wedge-shaped configuration (Figure 11) with a portion of the central foam's body removed and then tailored to allow the VPL placement into this patient's open abdomen between the anterior abdominal wall and the well-cocooned viscera. The VPL reconfiguring means that the blue foam encased in polyurethane was excised far back enough to remain covered by the polyurethan sleeve so as not to contact the viscera as specified in the ABTHERA ${ }^{\mathrm{TM}}$ directions which we agree and support. While recognized that this is not the manufacturer's preferred method for VPL placement as described in the manufacturer's directions, it may become necessary to "reimagine" the VPL to fit into this patient's abdominal domain. Using our simple modification techniques, surgeons can expand the use of NPWT temporary closure device deployments to include a larger population of various sized patients or those with complex abdomens without increased risk of morbidity.

\section{Conclusion}

Tailoring the VPL may be necessary when dealing with a complex, open abdomen that has adhesions or other intra-abdominal issues that limit the VPL's suc- 
cessful placement within the abdominal domain. Reimagining or being creative with the shape or configuration of the VPL while adhering to "no foam" exposure to the viscera may be necessary to affect a positive deployment of this device.

\section{Conflicts of Interest}

There are no conflicts of interest for the remaining authors of this manuscript.

\section{References}

[1] Miller, C. (2014) The History of Negative Pressure Wound Therapy (NPWT): From "Lip Service" to the Modern Vacuum System. Journal of the American College of Clinical Wound Specialists, 4, 61-62. https://doi.org/10.1016/j.jccw.2013.11.002

[2] Dirckx, J.H. (2008) Negative Pressure Wound Therapy. E-Perspectives, Health Professions Institute, 17-18.

[3] Fernandez, L.G., Sibaja Alvarez, P., Kaplan, M.J., Sanchez-Betancourt, A.A., Matthews, M.R. and Cook, A. (2019) Application of Negative Pressure Wound Therapy with Instillation and Dwell Time of the Open Abdomen: Initial Experience. Cureus, $11, \mathrm{e} 5667$.

[4] ABTHERA Active Abdominal Therapy. Product Specification Sheet. 3M/KCI Corporation (Minneapolis, $\mathrm{MN}$ ).

[5] (2018) ABTHERA ADVANCE ${ }^{\text {mi }}$ Open Abdomen Dressing. 1-7.

[6] Fernández, L.G. (2016) Management of the Open Abdomen: Clinical Recommendations for the Trauma/Acute Care Surgeon and General Surgeon. International Wound Journal, 3, 25-34. https://doi.org/10.1111/iwj.12655

[7] Fernandez, L.G. (2015) Temporary Abdominal Closure Techniques. http://emedicine.medscape.com/article/196820-overview

[8] Alvarez, P.S., Betancourt, A.S. and Fernández, L.G. (2012) Negative Pressure Wound Therapy with Instillation in the Septic Open Abdomen Utilizing a Modified Negative Pressure Therapy System. Annals of Medicine and Surgery, 36, 246-251. https://doi.org/10.1016/j.amsu.2018.10.007

[9] Sibaja Alvarez, P., Sánchez Betancourt, A. and Fernández, L.G. (2018) Negative Pressure Wound Therapy with Instillation in the Septic Open Abdomen Utilizing a Modified Negative Pressure Therapy System. Annals of Medicine and Surgery, 36, 246-251. https://linkinghub.elsevier.com/retrieve/pii/S2049080118302140 https://doi.org/10.1016/j.amsu.2018.10.007

[10] Matthews, M.R., Quan, A.N., Weir, A.S., Foster, K.N. and Caruso, D.M. (2018) Temporary Abdominal Closure Combined with an Irrigating System Utilizing Hypochlorous Acid Solution to Decrease Abdominal Mucopurulence. Eplasty, 18, e12.

[11] Fernández, L.G., Matthews, M.R. and Seal, L. (2020) Intraabdominal Lavage of Hypochlorous Acid: A New Paradigm for the Septic and Open Abdomen. Wounds. $A$ Compendium of Clinical Research and Practice, 32, 107-114.

[12] Betancourt, A.S., Milagros, G.C., Sibaja, P., Fernandez, L. and Norwood, S. (2020) Cost Evaluation of Temporary Abdominal Closure Methods in Abdominal Sepsis Patients Successfully Treated with an Open Abdomen. Should We Take Temporary Abdominal Closure Methods at Face Value? Health Economic Evaluation. Annals of Medicine and Surgery, 56, 11-16. https://doi.org/10.1016/j.amsu.2020.06.007 